\title{
Surgical Repair of a Superior Sternal Cleft in an Infant
}

\author{
Tobias Klein $^{1}$ Maximilian Kellner ${ }^{2}$ Thomas Michael Boemers $^{1}$ Birte Mack-Detlefsen ${ }^{1}$
}

1 Department of Pediatric Surgery and Pediatric Urology, Children's Hospital of Cologne, Cologne, Germany

2 Department of Pediatric Radiology, Children's Hospital of Cologne, Cologne, Germany

\begin{abstract}
Address for correspondence Tobias Klein, PA-C, Department of Pediatric Surgery and Pediatric Urology, Children's Hospital of Cologne, Amsterdamer Str. 59, Cologne, NRW 50735, Germany (e-mail: 7081.klein@gmail.com).
\end{abstract}

Eur J Pediatr Surg Rep 2015;3:64-67.
Abstract
Keywords
- sternal cleft
- chest wall malformation
- infant
- pericardial patch

Sternal cleft is a rare congenital malformation with little more than 100 cases published worldwide. Incomplete sternal clefting in a female newborn is the most frequent form seen. First-line treatment is the surgical defect closure in the neonatal period. Presurgical examination has to focus on common associated malformations, in particular cardiac defects. The surgical repair of sternal cleft itself shows satisfying functional and cosmetic results with low complication rates. We present the case of a 4-month-old male infant with a superior sternal cleft.

\section{New Insights and the Importance for the Pediatric Surgeon}

Sternal cleft is a rare congenital malformation that should be recognized by every pediatrician, pediatric surgeon and gynecologist, as its simple diagnosis can avert severe long-term consequences. Undiagnosed gas exchange impairment and respiratory dysfunction can result in i.a. respiratory distress and recurrent infections of the respiratory tract in the growing child. Pre-eminently the knowledge of its correlation with associated malformations like cardiac defects is of vital importance as specific diagnostics can be life-saving. By presenting our case of surgical repair of a superior sternal cleft in an infant we are able to recall our colleagues of this important malformation and issue instructions for diagnosis and surgical treatment.

\section{Introduction}

Sternal cleft (SC) is a rare congenital malformation that results from the defective embryologic fusion of mesenchymal cells in the ventral midline. The first case was reported in 1739 by De Torres. ${ }^{1}$ Since then data from little more than 100 cases have been published worldwide. Based on the clinical manifestation, the SC is classified into complete or incomplete, and the incomplete form being further subdivided in a superior and inferior type. At present 2 in 100,000 newborns are diagnosed with SC. ${ }^{2}$ In $67 \%$ of cases the SC is incomplete and two-thirds are female. Therefore, incomplete sternal clefting in a female is the more common anomaly. ${ }^{3}$

Associated malformations such as cardiovascular anomalies, midline fusion defects, hemangioma, maxillofacial defects, and pectus excavatus are common. Syndromic malformations coinciding with sternal clefting include PHACES (posterior fossa malformations-hemangiomas-arterial anomalies-cardiac defects-eye abnormalities-sternal cleft and supraumbilical raphe syndrome) or Cantrell pentalogy syndrome. $^{3}$

The fusion defect leaves the mediastinal viscera unprotected, resulting in an increased risk of harmful events to the underlying mediastinal organs. To restore the protective function of the skeleton by establishing bony integrity as soon as possible and to benefit from the high flexibility of the chest wall in newborns, surgical repair should be performed in the first weeks of life. ${ }^{3-5}$

Different surgical techniques such as primary closure, bone graft interposition, prosthetic closure, muscle flap interposition, and others have been described. ${ }^{3-8}$ received

November 11, 2014

accepted after revision

March 30, 2015

published online

June 15, 2015

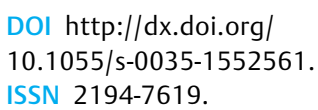

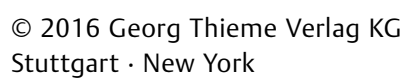

License terms

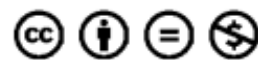


We present the case of a 4-month-old male infant with a superior SC.

\section{Case Report}

At 4 months, a male infant was referred to our department with the clinical diagnosis of a superior SC (-Fig. 1A). The SC was diagnosed postnatal by clinical examination in the maternity hospital and any associated cardiac anomalies had already been excluded. The clinical examination and ultrasound study (-Fig. 2) done in our hospital confirmed the diagnosis of SC and no further examinations were performed.

\section{Surgical Treatment}

After a vertical midline skin incision subcutaneous tissues were detached carefully. The two sternal bars were released from the underlying pericardium, thymus and mediastinal tissues and freed from the sternoclavicular joint (-Fig. 1B). A midline osteotomy was performed to separate the fused inferior part of the sternal bone. To facilitate closure of the SC wedge, excision at the bottom of the $\mathrm{V}$-formed defect was performed. A bovine pericardial patch (Peri-Guard, Synovis, St. Paul, Minnesota, United States,) was placed posteriorly before approximating the separated sternal bars in the midline applying PDS (polydioxanone) sutures (-Fig. 1C, D). A small residual gap was filled with fibrin glue and bone remnants collected during the osteotomy (-Fig. 1E). Wound closure was performed in two layers (-Fig. 1F). Neither a drain nor a chest tube was placed. Perioperative antibiotic treatment with cefuroxime was started and continued for 7 days.

On the first postoperative day the infant developed significant pleural effusion, and chest tubes were placed bilaterally in the intensive care unit (ICU). Additionally, a small pericardial effusion was present on the ultrasound examination. As a consequence diuretic treatment was started. However, the patient was successfully extubated on the forth postoperative day and complete oral intake was achieved the next day. On day 6 the chest tubes were removed since the pleural effusion

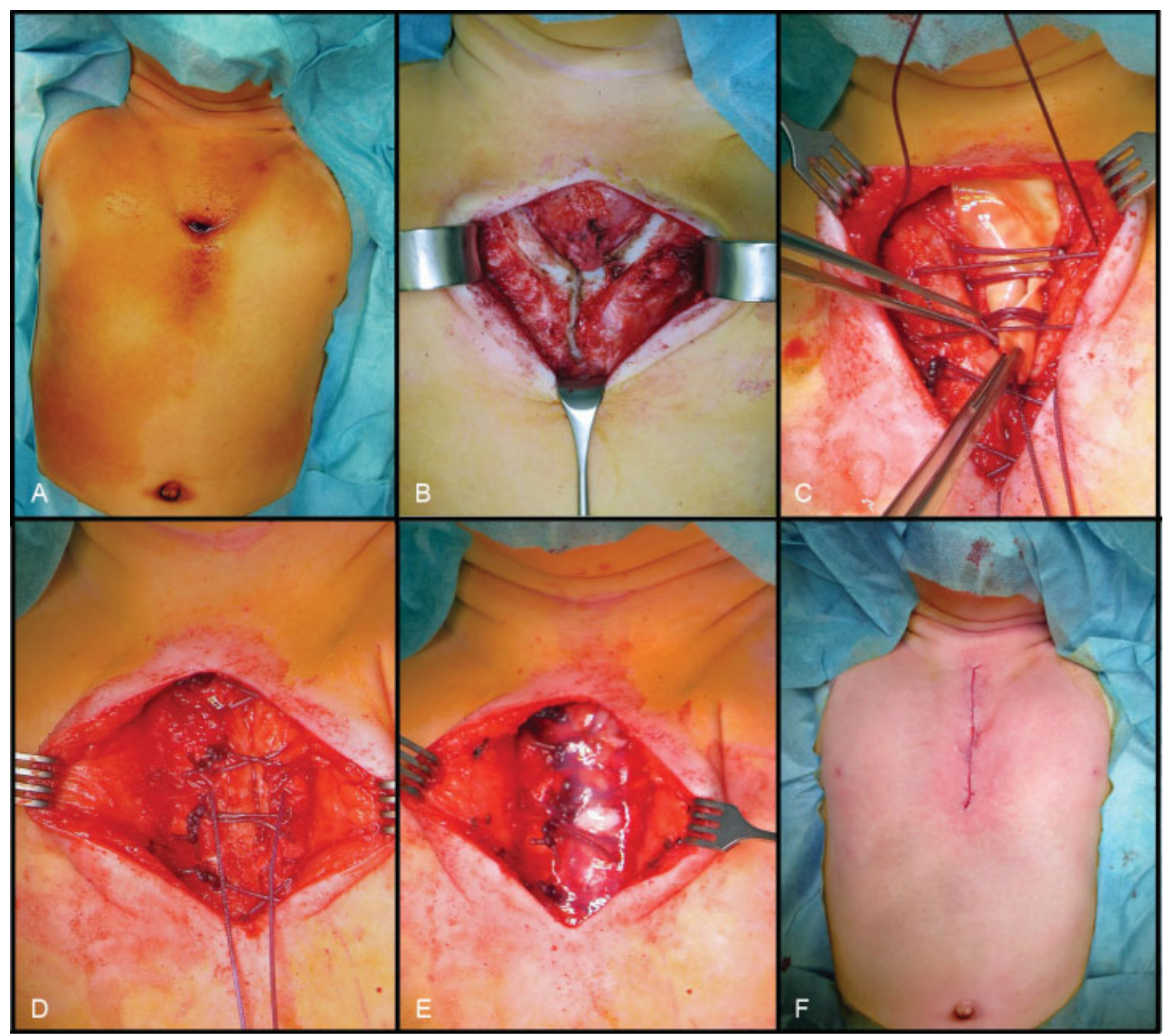

Fig. 1 Surgical closure of a superior sternal cleft in a 4-month-old male infant. (A) Preoperative. (B) SC after detachment of the subcutaneous tissue. (C) Placement of a bovine pericardial patch. (D) Approximation of the sternal bars. (E) Fibrin glue and bone insertion. (F) Postoperative result. 


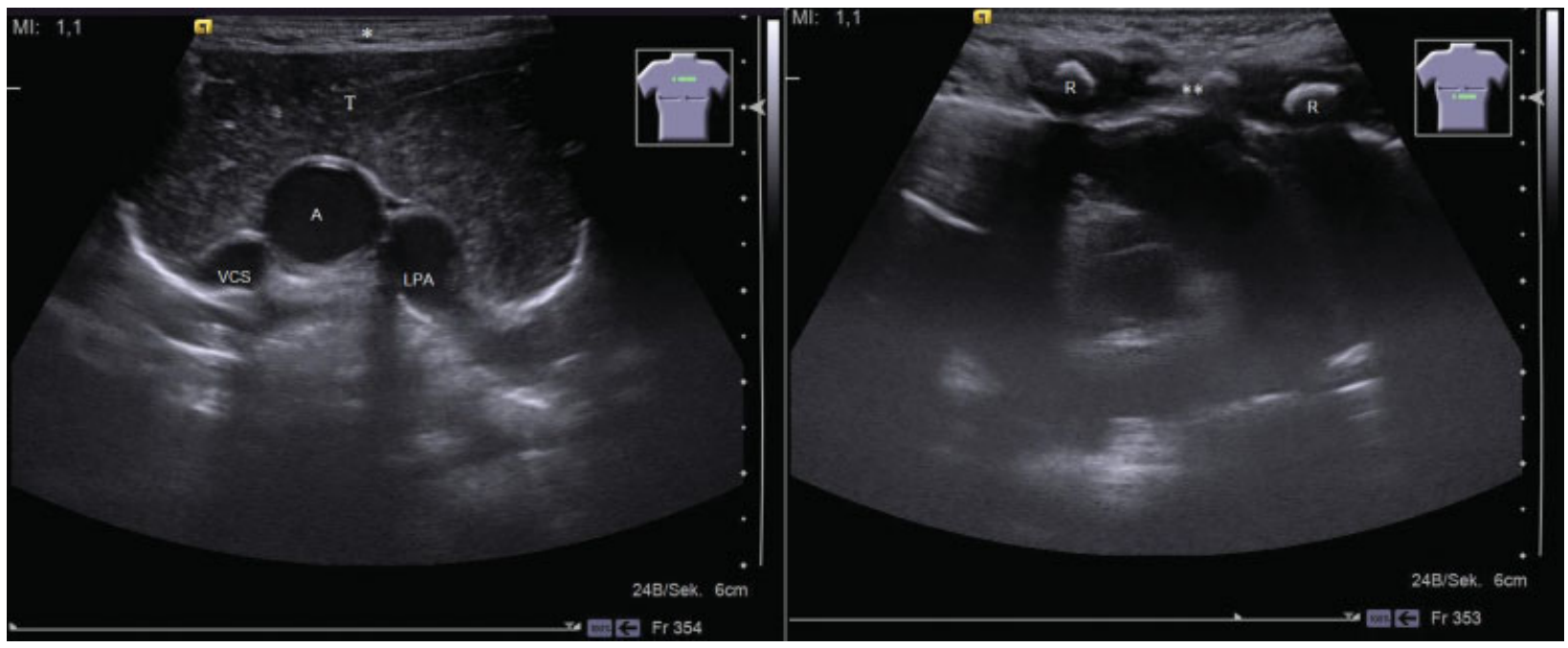

Fig. 2 Clinical examination and ultrasound study. A, ascending aorta; LPA, left pulmonary artery, R, ribs; T, thymus; VCS, vena cava superior. *Absence of superior part of the sternum. ${ }^{*}$ Regular inferior part of the sternum.

had resolved. The following clinical course passed without complications. The infant was discharged home on the 12th postoperative day.

The first postoperative checkup after 2 months and the long-term follow-up after 1 year showed very good cosmetic results and normal respiratory movements.

\section{Discussion}

$\mathrm{SC}$ is a rare congenital midline deformity of the chest wall. In regular embryologic development, two sternal bars deriving from the somatopleure start merging around the sixth week of gestation. ${ }^{9}$ The reason for fusion failure in SC is not yet known. A link to a group of genes that influence the body plan of the embryo along the anterior-posterior axis-the Hox genes-is being discussed. ${ }^{10}$

Usually sternal clefting is initially noticed in postnatal clinical examination by paradoxical respiratory movements in the newborn. Nevertheless some cases of late diagnosis in adolescence are described in the literature. ${ }^{4}$ Failure to diagnose the malformation in infants might be due to the absence of clinical symptoms in most newborns. ${ }^{3}$ If SC is not diagnosed and therefore not treated surgically in neonatal period, gas exchange impairment and respiratory dysfunction can result in dyspnea, respiratory distress, chronic coughing, or recurrent infections of the respiratory tract. ${ }^{3}$

Most patients with SC show associated malformations, in particular cardiac defects. ${ }^{11}$ Therefore ultrasound and cardiologic examinations including electrocardiogram (ECG) and echocardiography should be performed preoperatively. In our opinion radiologic imaging of the bony defect in conventional $\mathrm{X}$-rays or even computed tomographic (CT) scans, as postulated obligatory by some authors, ${ }^{3}$ is not necessary in most cases as no additional key information is gained and therefore radiation can be avoided. If associated malformations are suspected, further specific diagnostics are obligatory to prevent major complications during surgery. For example, if
PHACES syndrome is likely, magnetic resonance imaging (MRI) and ophthalmologic or neurologic workup should be done. Laryngotracheal bronchoscopy is mandatory to exclude a subglottic hemangioma in case of inspiratory stridor or patients presenting with other signs of hemangiomatosis.

The first report of a successful surgical SC repair in a 11-weekold child was published in 1947 by Burton. ${ }^{11}$ Prevailing opinion in the literature is that primary surgical closure performed in the neonatal age is the best treatment option., ${ }^{3,412}$ Because of the high flexibility of the chest wall of newborns, the primary closure is easier to perform and the risk for cardiovascular impairment is decreased. ${ }^{3,5}$ Nevertheless, it is critically important to monitor the hemodynamic and respiratory parameters closely during SC repair, as well as in the early postoperative phase. To this point different surgical techniques have been described in literature, including primary closure, sliding osteochondroplasty, and the use of periosteal or muscle flaps. ${ }^{3,4,8,13}$ In older patients or to treat larger defects, the use of autologous grafts or prosthetic material might be indicated as high pressure caused by reduced space and less compliance of the thoracic wall could severely impair mediastinal organ function. ${ }^{3,4}$ On the other hand, some authors claim the infection risk of artificial grafts might be increased and the missing adaption to ongoing growth could have negative impact on further development. ${ }^{4}$ In our patient a bovine patch was placed to create a second layer in the case of a diastasis of the two sternal bars postoperatively and to protect the underlying pleura. The postoperative course was complicated by development of a small pericardial effusion and pleural effusions, which had to be drained. We suggest that the pleural effusion results from a pleural tear during the preparation of the two sternal bars. In contrast to the complication seen in our case, the literature only reports the development of postoperative seromas. ${ }^{3,4}$ However, in different reports a drain was primarily placed to prevent fluid collections regardless of the use of prosthetic material., ${ }^{5,8}$ We didn't place drains to prevent infection such as mediastinitis in the postoperative course. In a review of the literature and according to his own 
case series, Torre et al found no negative effects on surgical outcome, stating that both techniques, primary closure as well as

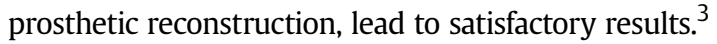

Throughout literature low complication rates are found. Intra- or postoperative mortality is rarely reported and seems to be related to associated malformations rather than to surgical intervention.

\section{Conclusion}

SC is a rare congenital chest malformation mostly diagnosed in newborns and infants. Primary surgical closure performed in the neonatal period or in early infancy is agreed upon as the therapy of choice. Ultrasound and cardiovascular examinations are obligatory eventually followed by complementary specific diagnostic procedures to rule out associated malformations prior to treatment. Overall the surgical repair of SC shows satisfying functional and cosmetic results with low complication rates.

\section{References}

1 De Torres JI. Extract of a letter from Jos. Ignat. De Torres, MD to the Royal Society, containing an extraordinary case of the heart of a child turned upside down. LondonPhilosophical Transactions1739;41:776-778

2 data PordB. Orphanet Report Series, Rare Diseases collection, No. 1: Listed in alphabetical order of disease or group of diseases. Available at: http://www.orpha.net/orphacom/cahiers/docs/GB/
Prevalence_of_rare_diseases_by_alphabetical_list.pdf. Accessed May 2014

3 Torre M, Rapuzzi G, Carlucci M, Pio L, Jasonni V. Phenotypic spectrum and management of sternal cleft: literature review and presentation of a new series. Eur J Cardiothorac Surg 2012;41(1):4-9

4 de Campos JR, Das-Neves-Pereira JC, Velhote MC, Jatene FB. Twenty seven-year experience with sternal cleft repair. Eur J Cardiothorac Surg 2009;35(3):539-541

5 Singh S, Lahoti BK, Garge S, Negi A, Jain V. Sternal cleft repair: a report of two cases and review of literature. Afr J Paediatr Surg 2010;7(3):211-213

6 Aggarwal M, Sarabahi S, Arya S, Kumar Tiwari V, Chellani H. Sternal cleft in a neonate-repaired with silastic block and pectoralis major flaps. Indian J Pediatr 2011;78(8):1013-1015

7 Baqain EB, Lataifeh IM, Khriesat WM, Fraiwan NM, Armooti MA. Primary repair of a large incomplete sternal cleft in an asymptomatic infant with Prolene mesh. J Pediatr Surg 2008;43(10): e39-e41

8 Muthialu N. Primary repair of sternal cleft in infancy using combined periosteal flap and sliding osteochondroplasty. Interact Cardiovasc Thorac Surg 2013;16(6):923-925

9 Engum SA. Embryology, sternal clefts, ectopia cordis, and Cantrell's pentalogy. Semin Pediatr Surg 2008;17(3):154-160

10 Forzano F, Daubeney PE, White SM. Midline raphé, sternal cleft, and other midline abnormalities: a new dominant syndrome? Am J Med Genet A 2005;135(1):9-12

11 Burton JF. Method of correction of ectopia cordis. Arch Surg 1947; 54(1):79-84

12 Acastello E, Majluf R, Garrido P, Barbosa LM, Peredo A. Sternal cleft: a surgical opportunity. J Pediatr Surg 2003;38(2):178-183

13 Snyder BJ, Robbins RC, Ramos D. Primary repair of complete sternal cleft with pectoralis major muscle flaps. Ann Thorac Surg 1996;61(3):983-984 